\title{
Myocardial apoptosis prevention by radical scavenging in patients undergoing cardiac surgery
}

\author{
Uwe M. Fischer, $\mathrm{MD}^{\mathrm{a}}$ \\ Paschalis Tossios, MD \\ Astrid Huebner, MD \\ Hans J. Geissler, MDa \\ Wilhelm Bloch, $\mathrm{PhD}^{\mathrm{b}}$ \\ Uwe Mehlhorn, MD ${ }^{\mathrm{a}}$
} From the Department of Cardiothoracic
Surgery ${ }^{\mathrm{a}}$ and Institute I for Anatomy, ${ }^{\mathrm{b}}$ Uni-
versity of Cologne, Cologne, Germany.
U.M.F. and P.T. contributed equally to this
work.
This work was supported by the German
Research Foundation (DFG: Me 1257/3-1).
Received for publication Aug 20, 2003; re-
visions requested Oct 10, 2003; revisions
received Nov 6, 2003; accepted for publi-
cation Dec 2, 2003.

Address for reprints: Uwe Mehlhorn, MD, Department of Cardiothoracic Surgery, University of Cologne, Joseph-StelzmannStr 9, 50924 Cologne, Germany (E-mail: uwe.mehlhorn@medizin.uni-koeln.de).

J Thorac Cardiovasc Surg 2004;128:103-8 $0022-5223 / \$ 30.00$

Copyright (C) 2004 by The American Association for Thoracic Surgery

doi:10.1016/j.jtcvs.2003.11.034
Background: Reactive oxygen-derived species, including those generated during myocardial ischemia and reperfusion induced by cardioplegia, have been suggested to be involved in myocardial apoptosis induction. The purpose of our study was to investigate (1) whether cardioplegic arrest initiates apoptosis in the hearts of cardiac surgery patients and (2) whether reactive oxygen-derived species scavenging with $\mathrm{N}$-acetylcysteine attenuates myocardial apoptosis initiation.

Methods: In transmural left ventricular biopsy samples collected before and at the end of cardiopulmonary bypass, we densitometrically determined cardiac myocyte staining intensity for active caspases-3 and -7 , the apoptosis signal pathway central effector enzymes. The left ventricular biopsy samples had been obtained from 36 coronary artery bypass graft patients randomized in a double-blind fashion to receive either $N$-acetylcysteine $(100 \mathrm{mg} / \mathrm{kg}$ into cardiopulmonary bypass prime followed by infusion at $\left.20 \mathrm{mg} \cdot \mathrm{kg}^{-1} \cdot \mathrm{h}^{-1} ; \mathrm{n}=18\right)$ or placebo $(\mathrm{n}=18)$.

Results: The change in left ventricular cardiac myocyte staining (end of cardiopulmonary bypass minus before cardiopulmonary bypass) differed significantly between groups for both measures: caspase- $3,-3.1 \pm 4.5$ gray units (mean $\pm \mathrm{SD}$; $\mathrm{N}$-acetylcysteine group) versus $7.1 \pm 8.1$ gray units (placebo); $95 \%$ confidence interval, 6.4 to $14.4 ; P<.0001$; caspase- $7,-5.1 \pm 6.1$ gray units ( $N$-acetylcysteine) versus $5.1 \pm 5.7$ gray units (placebo); $95 \%$ confidence interval, 6.3 to $15.0 ; P<$ .0001 . Clinical outcome did not differ between $N$-acetylcysteine and placebo.

Conclusions: Our data show that cardioplegic arrest initiates the apoptosis signal cascade in human left ventricular cardiac myocytes. This apoptosis induction can effectively be prevented by $\mathrm{N}$-acetylcysteine.

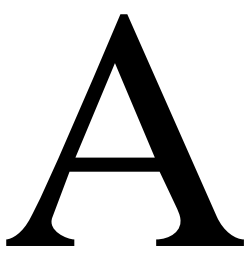

poptosis has been shown to be involved in various cardiac pathologies, including heart failure, cardiomyopathy, myocarditis, and myocardial ischemia/reperfusion. ${ }^{1-3}$ Apoptosis can be triggered through different mechanisms in response to both intracellular and extracellular signals. ${ }^{4,5}$ For example, cytokine release by macrophages and myocytes can activate the apoptosis signal cascade. ${ }^{6}$ In addition, reactive oxygen-derived species (ROS), often called oxidative stress, have been shown to induce apoptosis in cardiac myocytes subjected to ischemia and reperfusion. ${ }^{7,8}$ Accordingly, we and other researchers have recently demonstrated that cardioplegia-induced ischemia and reperfusion is associated with apoptosis initiation in adult porcine, ${ }^{9}$ canine, ${ }^{10}$ and rat $^{11}$ and neonatal lamb left ventricular (LV) myocytes, ${ }^{12}$ as well as human atrial myocytes. ${ }^{13}$ Because cardiac apoptosis has been suggested to be involved in postoperative myocardial stunning, ${ }^{13}$ cardiac apoptosis prevention could represent an attractive adjunct to current myocardial protection techniques in cardiac surgery. 
TABLE 1. Baseline demographic and clinical characteristics of trial groups ${ }^{14}$

\begin{tabular}{|c|c|c|}
\hline Characteristic & $\begin{array}{l}\text { NAC group } \\
(n=20)\end{array}$ & $\begin{array}{l}\text { Placebo group } \\
\quad(\mathrm{n}=\mathbf{2 0})\end{array}$ \\
\hline Age, y (mean $\pm S D)$ & $66.2 \pm 11.8$ & $66.5 \pm 6.5$ \\
\hline Female, $\mathrm{n}(\%)$ & $8(40)$ & $1(5)$ \\
\hline $\begin{array}{l}\text { Arterial hypertension, } \\
\mathrm{n}(\%)\end{array}$ & 17 (85) & $16(80)$ \\
\hline Diabetes, n (\%) & $7(35)$ & $6(30)$ \\
\hline Smoking history, n (\%) & $9(45)$ & $7(35)$ \\
\hline $\begin{array}{l}\text { Previous myocardial } \\
\text { infarction, } n(\%)\end{array}$ & $6(30)$ & $10(50)$ \\
\hline \multicolumn{3}{|l|}{ Vessel disease, $\mathrm{n}(\%)$} \\
\hline 1 & $1(5)$ & $0(0)$ \\
\hline 2 & $3(15)$ & $4(20)$ \\
\hline 3 & $16(80)$ & $16(80)$ \\
\hline $\begin{array}{l}\text { Body mass index, kg/ } \\
\mathrm{m}^{2}(\text { mean } \pm S D)\end{array}$ & $27.3 \pm 4.4$ & $27.8 \pm 3.7$ \\
\hline $\begin{array}{l}\text { Angiographic LV } \\
\text { ejection fraction, \% } \\
\text { (mean } \pm \text { SD) }\end{array}$ & $57.9 \pm 16.7(\mathrm{n}=17)^{*}$ & $63.9 \pm 14.1(\mathrm{n}=17)^{*}$ \\
\hline $\begin{array}{l}\text { LVEDP, mm Hg (mean } \\
\quad \pm \mathrm{SD} \text { ) }\end{array}$ & $15.5 \pm 8.3(n=15)^{*}$ & $14.8 \pm 6.7(n=13)^{*}$ \\
\hline $\begin{array}{l}\text { Cardiac index, } \mathrm{L} \text {. } \\
\quad \min ^{-1} \cdot \mathrm{L}^{-2} \text { (mean } \\
\quad \pm \mathrm{SD})\end{array}$ & $2.47 \pm 0.45(n=19) \dagger$ & $2.55 \pm 0.47$ \\
\hline $\mathrm{FAC}, \%$ (mean $\pm \mathrm{SD}$ ) & $63.1 \pm 11.0(n=18) \ddagger$ & $61.0 \pm 11.3(n=17) \ddagger$ \\
\hline
\end{tabular}

NAC, $\mathrm{N}$-Acetylcysteine; $L V E D P$, left ventricular end-diastolic pressure; $F A C$, fractional area of contraction as determined by transesophageal echocardiography before cardiopulmonary bypass.

Reasons for missing values ( $\mathrm{n}<20$ ) are ${ }^{*}$ not determined at the time of heart catheter insertion; tcatheter placement impossible; and ftransesophageal echocardiography not performed.

One potential apoptosis prevention strategy could be ROS scavenging. Experimentally, Dobsak and colleagues ${ }^{11}$ have recently shown that ROS scavenging with the iron chelator deferoxamine resulted in fewer apoptotic myocytes associated with better functional recovery in rat hearts after 4 hours of cardioplegic arrest. We have recently demonstrated that the antioxidant and ROS scavenger $\mathrm{N}$-acetylcysteine (NAC) prevented direct ROS-mediated myocardial alterations in the LV myocardium of patients subjected to cardioplegia $^{14}$; however, whether NAC attenuates cardiac apoptosis initiation has not been investigated.

Therefore, the purpose of our study was to compare the effects of NAC versus placebo on activation of caspases- 3 and -7 , the apoptosis signal pathway central effector enzymes, in LV myocardium of patients subjected to coronary artery operation during cardioplegic arrest.

\section{Materials and Methods}

After approval by the University of Cologne Human Ethics Committee, written, informed consent was obtained from each patient during the preoperative interview. We used LV biopsy specimens from our previous study, ${ }^{14}$ in which we randomized 40 patients (9 women and 31 men) scheduled for elective or urgent coronary artery bypass surgery into either the NAC group $(n=20)$ or the placebo group $(n=20)$ according to a computer-generated allocation list. Patients' biometric data and procedures are given in Tables 1 and 2 according to reference. ${ }^{14}$ Briefly, patients of the NAC group received $100 \mathrm{mg}$ of NAC per kilogram body weight into the cardiopulmonary bypass $(\mathrm{CPB})$ prime followed by intravenous infusion at $20 \mathrm{mg}$ of NAC per kilogram of body weight per hour until the end of CPB; patients in the placebo group received equivalent amounts of placebo. Patients were subjected to $\mathrm{CPB}$ at $32^{\circ} \mathrm{C}$ to $34^{\circ} \mathrm{C}$, the aorta was crossclamped, and myocardial revascularization was performed during cardioplegic arrest by using single-shot antegrade cold $\left(4^{\circ} \mathrm{C}\right)$ crystalloid Bretschneider cardioplegia (Custodiol; Dr Köhler Chemie, Alsbach, Germany).

\section{Biopsies}

Before CPB initiation, we collected a transmural biopsy sample from a fat-free area of the LV anterior wall by using a 14-gauge biopsy needle (Gallini, Modena, Italy). A second LV biopsy sample was taken at the end of the extracorporeal circulation before weaning from CPB. All LV biopsy samples were placed in $4 \%$ paraformaldehyde for 4 hours and then rinsed in $0.1 \mathrm{~mol} / \mathrm{L}$ phosphate-buffered saline (PBS) for 24 hours, followed by storage for 12 hours in PBS solution with $18 \%$ sucrose for cryoprotection. Finally, they were frozen at $-80^{\circ} \mathrm{C}$. Because of the small size of the LV biopsy samples, we were able to densitometrically quantitate cardiac myocyte staining for active caspase- 3 (before CPB and at the end of $\mathrm{CPB}$ ) in 18 patients of the placebo group and 18 patients of the NAC group and for active caspase-7 in 17 patients of the placebo group and 17 patients of the NAC group.

\section{Immunocytochemistry}

Before immunohistochemical examination, 7- $\mu \mathrm{m}$ slices from the biopsy samples were placed in a bathing solution of $3 \% \mathrm{H}_{2} \mathrm{O}_{2}$ and methanol for 20 minutes, and then cells were lysed with $0.25 \%$ Triton X-100 (Rohm \& Haas Co, Philadelphia, Pa) in ammonium chloride $0.5 \mathrm{~mol} / \mathrm{L}$. Thereafter, specimens were treated with $5 \%$ bovine serum albumin solution in Tris-buffered saline (TBS) 0.05 $\mathrm{mol} / \mathrm{L}$. Before each step, the sections were rinsed 3 times in TBS buffer $0.05 \mathrm{~mol} / \mathrm{L}$. Incubation with primary rabbit anti-active caspase-3 antibody (1:500; Pharmingen, San Diego, Calif) and rabbit anti-active caspase-7 antibody (1:500; Biocat, Heidelberg, Germany) were performed in a TBS-based solution of $0.8 \%$ bovine serum albumin and $\mathrm{NaN}_{3} 20 \mathrm{mmol} / \mathrm{L}$ for 12 hours at $4^{\circ} \mathrm{C}$. After rinsing with TBS, the sections were incubated with the corresponding secondary biotinylated goat anti-rabbit antibody (1:400; DAKO, Hamburg, Germany) for 1 hour at room temperature. A streptavidin-horseradish-peroxidase complex was then applied as a detection system (1:150) for 1 hour. Finally, staining was developed for 10 to 20 minutes with 3,3-diaminobenzidine tetrahydrochloride in PBS $0.1 \mathrm{~mol} / \mathrm{L}$.

Active Caspase-3 and Caspase-7 Television Densitometry All LV biopsy slices were incubated and stored under identical conditions. For quantitative intensity analyses of active caspase-3 and -7 immunostaining in cardiac myocytes, we measured the gray values of 30 cardiac myocytes from 6 randomly selected areas. The staining intensity was reported as the mean measured cardiac 
TABLE 2. Perioperative characteristics and post-cardiopulmonary bypass hemodynamics of trial groups ${ }^{14}$

\begin{tabular}{|c|c|c|}
\hline Characteristic & $\begin{array}{c}\text { NAC group } \\
(\mathbf{n}=\mathbf{2 0})\end{array}$ & $\begin{array}{c}\text { Placebo group } \\
(\mathbf{n}=\mathbf{2 0 )}\end{array}$ \\
\hline \multicolumn{3}{|l|}{ Grafts, n (\%) } \\
\hline 1 & $1(5)$ & $0(0)$ \\
\hline 2 & $1(5)$ & $5(25)$ \\
\hline 3 & $10(50)$ & $9(45)$ \\
\hline 4 & $7(35)$ & $5(25)$ \\
\hline 5 & $1(5)$ & 1 (5) \\
\hline ITA use, n (\%) & $20(100)$ & $18(90)$ \\
\hline Hospital mortality, n (\%) & $0(0)$ & $0(0)$ \\
\hline Perioperative myocardial infarction, $\mathrm{n}(\%)$ & $0(0)$ & $0(0)$ \\
\hline \multicolumn{3}{|l|}{ Duration, min (mean $\pm S D)$} \\
\hline Operation & $175 \pm 25$ & $157 \pm 20$ \\
\hline Cardiopulmonary bypass & $80 \pm 19$ & $73 \pm 18$ \\
\hline Aortic crossclamp & $43 \pm 10$ & $38 \pm 9$ \\
\hline Reperfusion & $28 \pm 7$ & $27 \pm 8$ \\
\hline Dopamine, $\mu \mathrm{g} \cdot \mathrm{kg}^{-1} \cdot \min ^{-1}($ mean $\pm \mathrm{SD})$ & $4.2 \pm 1.6$ & $4.2 \pm 1.4$ \\
\hline Postoperative CK, U/L (mean \pm SD) & $84.8 \pm 26.3$ & $86.6 \pm 23.3(n=19)^{*}$ \\
\hline Postoperative CK-MB, U/L (mean \pm SD) & $13.0 \pm 3.7$ & $10.3 \pm 3.9(\mathrm{n}=19)^{*}$ \\
\hline Heart rate, beats/min (mean $\pm S D$ ) & $91.2 \pm 10.7$ & $88.5 \pm 12.9$ \\
\hline Arterial blood pressure, $\mathrm{mm} \mathrm{Hg}$ (mean $\pm \mathrm{SD}$ ) & $69.0 \pm 8.5$ & $70.0 \pm 9.2$ \\
\hline $\mathrm{PAP}, \mathrm{mm} \mathrm{Hg}($ mean $\pm \mathrm{SD})$ & $15.7 \pm 5.8(n=19) \dagger$ & $14.1 \pm 4.0$ \\
\hline PCWP, mm Hg (mean \pm SD) & $9.8 \pm 5.3(\mathrm{n}=19) \dagger$ & $9.7 \pm 3.9(n=19) \ddagger$ \\
\hline CVP, $\mathrm{mm} \mathrm{Hg}$ (mean $\pm \mathrm{SD})$ & $7.8 \pm 3.9(n=19) \dagger$ & $7.0 \pm 4.3$ \\
\hline SVRI, dyne $\cdot \mathrm{s}^{-1} \cdot \mathrm{m}^{-2} \cdot \mathrm{cm}^{-5}($ mean $\pm \mathrm{SD})$ & $1516 \pm 471(\mathrm{n}=19) \dagger$ & $1325 \pm 256$ \\
\hline PVRI, dyne $\cdot \mathrm{s}^{-1} \cdot \mathrm{m}^{-2} \cdot \mathrm{cm}^{-5}($ mean $\pm \mathrm{SD})$ & $143 \pm 75(n=19) \dagger$ & $110 \pm 49(\mathrm{n}=18) \S$ \\
\hline
\end{tabular}

ITA, Internal thoracic artery; PAP, pulmonary artery pressure; $P C W P$, pulmonary capillary wedge pressure; $C V P$, central venous pressure; SVRI, systemic vascular resistance index; $P V R I$, pulmonary vascular resistance index; $C K$, creatine kinase; $C K-M B$, creatine kinase myocardial band; $N A C, N$-acetylcysteine.

Reasons for missing values $(\mathrm{n}<20)$ are *data not in patient chart; tno pulmonary artery catheter; łno wedge position of the pulmonary artery catheter balloon; §calculation not possible because of $\ddagger(n=1)$ and $n=1$ was excluded because of a negative value (PAP $<$ PCWP).

myocyte gray value minus the background gray value. The background gray value was measured at a cell-free area of the slice. For staining intensity detection, a Zeiss (Jena, Germany) Axiophot microscope coupled to a 3-chip charge-coupled device camera was used, and analysis was performed by using the Optimas 6.01 image-analysis program (Optimas 6.01, Media Cybernetics, Silver Spring, Md) installed on a personal computer.

\section{Statistical Analysis}

Continuous variables were summarized as mean \pm SD. Changes in outcome variables were analyzed for statistical significance at a level of $\alpha=5 \%$ by using 2-tailed Student $t$ tests for unpaired or paired samples, where appropriate. Corresponding $95 \%$ confidence intervals (CI) are given to allow assessment of effect sizes for clinical relevance. Statistical analyses were performed with the software package SPSS for Windows, release 10.0.7 (SPSS Inc, Chicago, Ill). The $P$ values reported were not adjusted for multiple testing.

\section{Results}

Cardiac myocyte immunostaining for active caspases- 3 and -7 is depicted in Figure 1. Compared with before CPB, LV cardiac myocytes of the placebo group demonstrated caspase- 3 and -7 activation at the end of CPB. In contrast, cardiac myocytes of the NAC group remained negative for both caspases at the end of CPB. In the placebo group, caspase-3 staining increased from $11.6 \pm 2.4$ gray units before CPB to $18.7 \pm 8.0$ gray units at the end of CPB ( $P$ $=.0017 ; 95 \% \mathrm{CI}$ for mean change, 3.4 to 10.9$)$, and caspase-7 staining increased from $23.8 \pm 11.2$ gray units before CPB to $28.9 \pm 12.0$ gray units at the end of CPB $(P$ $=.0019 ; 95 \% \mathrm{CI}$ for mean change, 2.4 to 7.8$)$. In the NAC group, caspase-3 staining decreased from $14.9 \pm 3.4$ gray units before CPB to $11.7 \pm 2.6$ gray units at end of CPB ( $P$ $=.009 ; 95 \% \mathrm{CI}$ for mean change, -1.1 to -5.2$)$, and caspase-7 staining decreased from $25.8 \pm 11.0$ gray units before CPB to $20.8 \pm 11.3$ gray units at the end of CPB ( $P$ $=.003 ; 95 \% \mathrm{CI}$ for mean change, -2.2 to -8.0$)$. The changes (from before $\mathrm{CPB}$ to the end of $\mathrm{CPB}$ ) in cardiac myocyte density for caspases-3 and -7 are depicted in Figure 2.

Patients' biometrics, intraoperative procedures, and clinical outcomes were similar between groups; there were no deaths or perioperative myocardial infarctions ${ }^{14}$ (Tables 1 and 2). We did not observe adverse effects attributable to NAC. Post-CPB hemodynamics, including heart rate, vas- 


\section{active caspase-3}
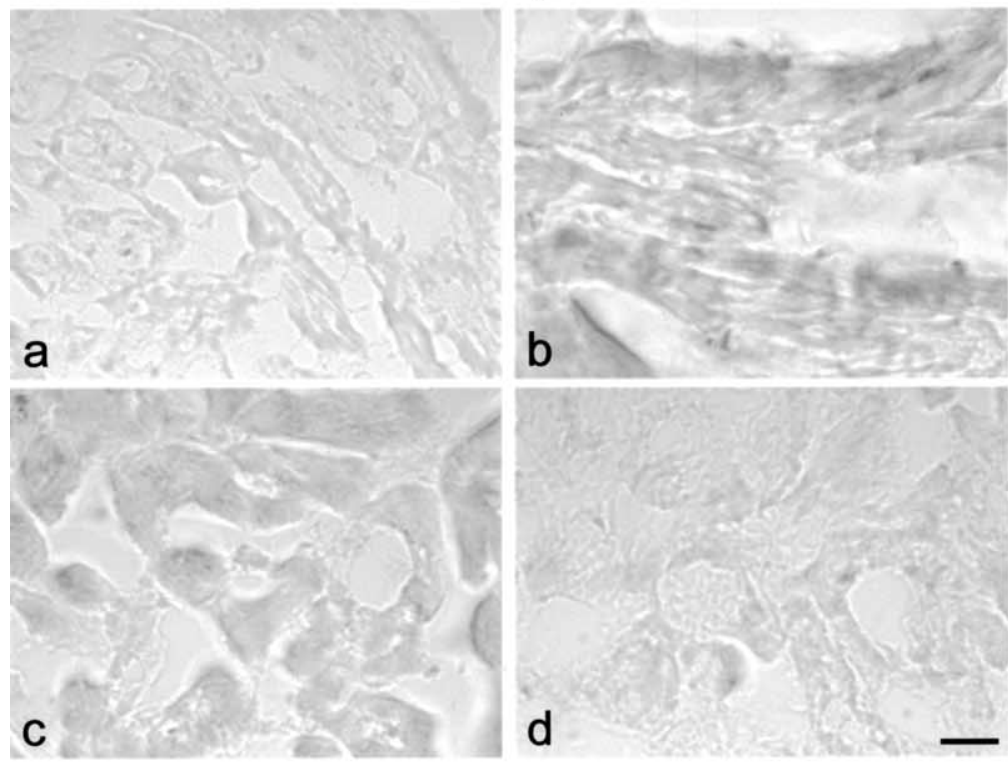

\section{active caspase-7}

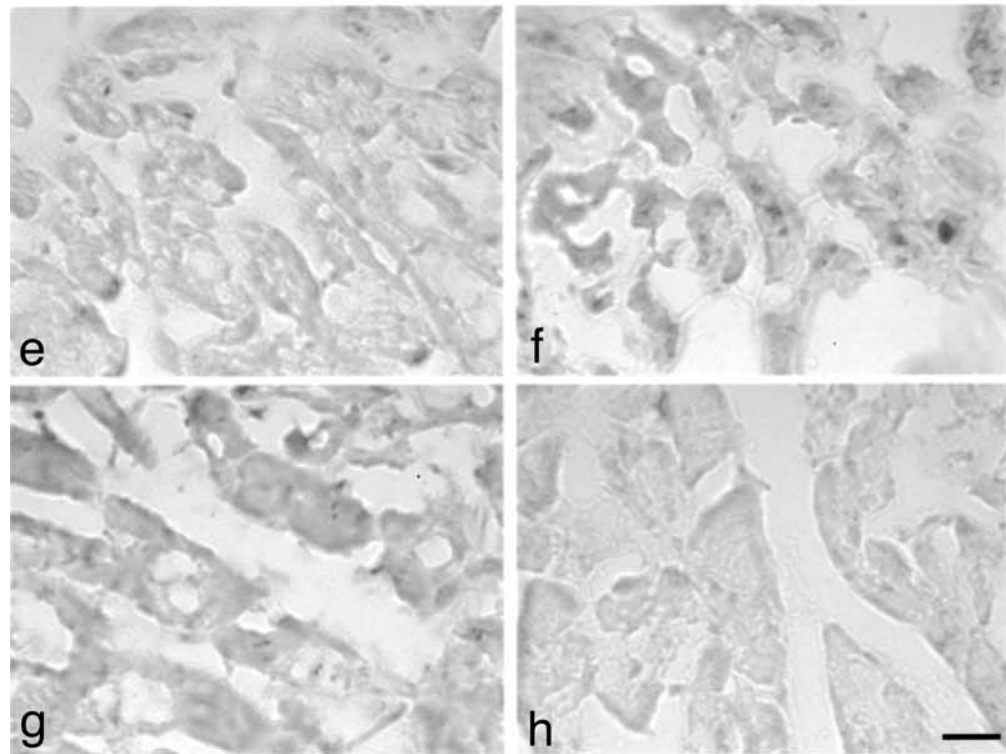

Figure 1. Immunostaining for active caspases-3 and -7 in cardiac myocytes and capillaries shows that compared with before CPB (a and e), LV cardiac myocytes of the placebo group demonstrated caspase-3 (b) and caspase-7 (f) activation at the end of CPB. In contrast, cardiac myocytes of the NAC group, even if slightly stained before CPB (c and g), were negative for both active caspase-3 (d) and active caspase-7 (h) at the end of CPB (bar $=35 \mu \mathrm{m}$ ).

cular pressures, systemic and pulmonary vascular resistance, cardiac index, LV function as measured by transesophageal echocardiography, and positive inotropic medication, were similar between groups ${ }^{14}$ (Tables 1 and 2). We did not find differences among the degree of caspase staining and arterial hypertension, diabetes mellitus, LV ejection fraction, or LV end-diastolic pressure $(P>.27)$.

\section{Discussion}

Our data show that NAC prevents cardioplegia-induced apoptosis signal cascade initiation in human LV myocardium, because both cardiac myocyte caspase-3 and caspase-7 activities were significantly lower in patients who received NAC compared with placebo. Together with our clinical trial, which demonstrated that NAC protected the 
myocardium from direct ROS-mediated alterations such as 8 -iso-prostaglandin- $\mathrm{F}_{2} \alpha$ and nitrotyrosine formation, ${ }^{14}$ these data show for the first time that ROS scavenging can effectively attenuate cardiac myocyte apoptosis induction in patients who undergo cardioplegia.

Apoptosis is a genetically programmed process for the death and subsequent removal of injured cells. Several extracellular and intracellular stimuli, including cytokine release, mechanical stretch, and oxidative stress, can trigger the apoptosis signal cascade. ${ }^{4-6}$ The apoptosis execution phase is initiated by cleavage and, thus, activation of downstream or effector caspases such as caspases-3 and $-7 ., 4$ Subsequently, these activated caspases can cleave genomic DNA, leading to DNA fragmentation and, ultimately, apoptotic cell death. ${ }^{4,5}$ Even though the clinical relevance of cardioplegia-induced apoptosis is not yet well established, recent work suggests that apoptosis is initiated by cardioplegic arrest in diseased adult hearts and may contribute to postoperative myocardial stunning. ${ }^{13}$ It has to be noted, however, that detection of cleaved caspases as performed in this study does not necessarily indicate substrate cleavage by activated caspases, because inhibitory proteins may block cleaved caspases. In fact, this inhibition of the apoptosis signal cascade explains the unchanged post-CPB cardiac function without signs of massive apoptotic cardiac cell loss despite the homogeneous myocardial apoptosis initiation induced by cardioplegia and reperfusion. However, considering the potential long-term effects attributable to apoptosis initiation, including mitochondrial alterations, functional and structural protein derangement, and accelerated cell aging, ${ }^{4,5,15}$ apoptosis prevention has to be regarded as a cardioprotective measure.

Because ROS can trigger apoptosis ${ }^{4,5,7-9}$ and because cardioplegia-induced myocardial ischemia/reperfusion is associated with massive ROS production, ${ }^{16}$ ROS scavenging may attenuate apoptosis induction in hearts exposed to cardioplegia. Experimentally, effective apoptosis prevention by ROS scavenging with the glutathione peroxidase mimic ebselen has been demonstrated in a clinically relevant pig model of regional myocardial ischemia and cardioplegic arrest. ${ }^{17}$ In addition, ROS scavenging with the iron chelator deferoxamine resulted in fewer apoptotic myocytes and better functional recovery in rat hearts after 4 hours of cardioplegic arrest. ${ }^{11}$ In this study, we found that the antioxidant and reduced glutathione precursor NAC prevented the activation of caspases- 3 and -7 in the LV myocytes of patients subjected to cardioplegia. In contrast, the hearts of patients in the placebo group demonstrated significant caspase- 3 and -7 activation, indicating apoptosis signal cascade initiation. Although the early postoperative hemodynamics and short-term clinical outcome were not different between the NAC and placebo groups, ${ }^{14}$ these data

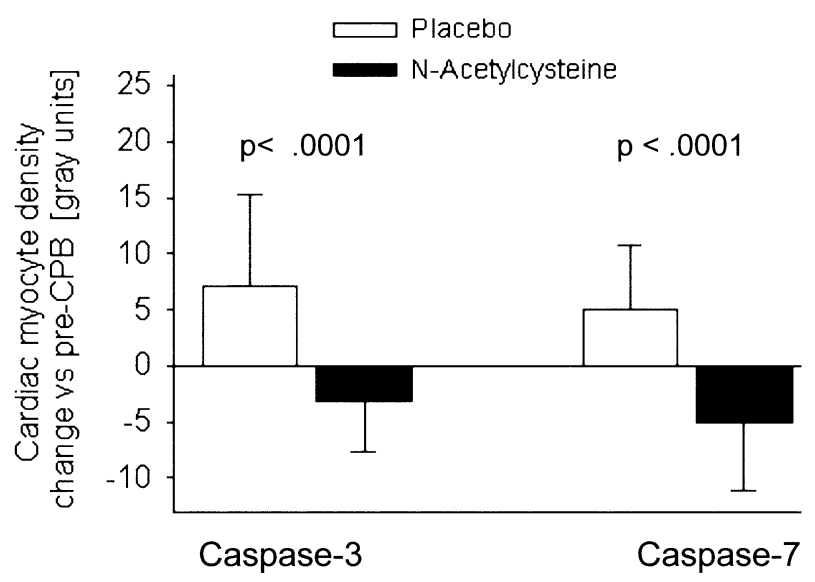

Figure 2. Change from before CPB to the end of CPB in cardiac myocyte density for active caspases- 3 and -7 in both groups (data are mean $\pm S D$; for caspase-3, $n=18$ in the placebo group; $n=$ 18 in the $\mathrm{N}$-acetylcysteine group; for caspase-7, $\mathrm{n}=17$ in the placebo group; $\mathbf{n}=17$ in the $\mathbf{N}$-acetylcysteine group).

suggest that ROS scavenging with NAC may be a useful adjunct to myocardial protection strategies.

It is interesting that we found that in the NAC group, cardiac myocyte caspase-3 and -7 activities actually decreased from before $\mathrm{CPB}$ to the end of $\mathrm{CPB}$, indicating apoptosis signal cascade activation during the period of anesthesia induction, thoracotomy, and cannulation for CPB. Because previous work showed myocardial 8-isoprostaglandin- $\mathrm{F}_{2} \alpha$ and nitrotyrosine formation before $\mathrm{CPB},{ }^{16}$ some ROS must have been induced by anesthesia induction, surgical trauma, or a combination thereof. Thus, to yield the full potential benefit of its ROS-scavenging properties, NAC application should start before anesthesia induction.

In conclusion, our data show for the first time that cardioplegia-induced apoptosis signal cascade activation in human LV cardiac myocytes can effectively be prevented by ROS scavenging with NAC. However, the data of our study do not allow us to determine how many cardiac myocytes, if any, completed the apoptosis signal cascade in hearts not protected by NAC, because apoptosis completion probably requires several hours ${ }^{18}$ and, thus, cannot be detected within the time frame of routine coronary artery surgery. Despite this lack of evidence for apoptosis completion, caspase activity may still have a significant effect on myocardial function. As shown by Ricci and colleagues, ${ }^{19}$ caspase- 3 cleavage is also associated with functional deficits of complex I and complex II in the electron transport chain when the outer mitochondrial membrane has been permeabilized, as occurs upstream of caspase activation. Therefore, caspase activation inhibition seems to be beneficial even before apoptosis is completed. Future studies are required to further elucidate the clinical relevance of 
cardioplegia-induced cardiac myocyte apoptosis, its regulation, and the time course of apoptosis completion.

\section{References}

1. Olivetti G, Abbi R, Quaini F, Kajstura J, Cheng W, Nitahara JA, et al. Apoptosis in the failing human heart. N Engl J Med. 1997;336:113141.

2. Saraste A, Pulkki K, Kallajoki M, Henriksen K, Parvinen M, VoipioPulkki LM. Apoptosis in human acute myocardial infarction. Circulation. 1997;95:320-3.

3. Narula J, Haider N, Virmani R, DiSalvo TG, Kolodgie FD, Hajjar RJ, et al. Apoptosis in myocytes in end-stage heart failure. $N$ Engl J Med. 1996:335:1182-9.

4. Haunstetter A, Izumo S. Apoptosis: basic mechanisms and implications for cardiovascular disease. Circ Res. 1998;82:1111-29.

5. Dispersyn GD, Borgers M. Apoptosis in the heart: about programmed cell death and survival. News Physiol Sci. 2001;16:41-7.

6. Gurevitch J, Frolkis I, Yuhas Y, Paz Y, Matsa M, Mohr R, et al. Tumor necrosis factor-alpha is released from the isolated heart undergoing ischemia and reperfusion. J Am Coll Cardiol. 1996;28:247-52.

7. von Harsdorf R, Li P-F, Dietz R. Signaling pathways in reactive oxygen species-induced cardiomyocyte apoptosis. Circulation. 1999; 99:2934-41.

8. Droege W. Free radicals in the physiological control of cell function. Physiol Rev. 2002;82:47-95.

9. Fischer UM, Klass O, Stock U, Easo J, Geissler HJ, Fischer JH, et al. Cardioplegic arrest induces apoptosis signal-pathway in myocardial endothelial cells and cardiac myocytes. Eur J Cardiothorac Surg. 2003;23:984-90

10. Yeh CH, Wang YC, Wu YC, Chu JJ, Lin PJ. Continuous tepid blood cardioplegia can preserve coronary endothelium and ameliorate the occurrence of cardiomyocyte apoptosis. Chest. 2003;123:1647-54.
11. Dobsak P, Siegelova J, Wolf JE, Rochette L, Eicher JC, Vasku J, et al. Prevention of apoptosis by deferoxamine during 4 hours of cold cardioplegia and reperfusion: in vitro study of isolated working rat heart model. Pathophysiology. 2002;9:27-32.

12. Hammel JM, Caldarone CA, Van Natta TL, Wang LX, Welke KF, Li $\mathrm{W}$, et al. Myocardial apoptosis after cardioplegic arrest in the neonatal lamb. J Thorac Cardiovasc Surg. 2003;125:1268-75.

13. Schmitt JP, Schroder J, Schunkert H, Birnbaum DE, Aebert H. Role of apoptosis in myocardial stunning after open heart surgery. Ann Thorac Surg. 2002;73:1229-35.

14. Tossios P, Bloch W, Huebner A, Raji MR, Dodos F, Klass O, et al. $\mathrm{N}$-Acetylcysteine prevents reactive oxygen species-mediated myocardial stress in cardiac surgery patients: results of a randomized doubleblinded placebo-controlled clinical trial. J Thorac Cardiovasc Surg. 2003;126:1513-20.

15. Pollack M, Phaneuf S, Dirks A, Leeuwenburgh C. The role of apoptosis in the normal aging brain, skeletal muscle, and heart. Ann NY Acad Sci. 2002;959:93-107.

16. Mehlhorn U, Krahwinkel A, Geissler HJ, Larosee K, Fischer UM, Klass O, et al. Nitrotyrosine and 8-isoprostane formation indicate free radical-mediated injury in hearts of patients subjected to cardioplegia. J Thorac Cardiovasc Surg. 2003;125:178-83.

17. Maulik N, Yoshida T. Oxidative stress developed during open heart surgery induces apoptosis: reduction of apoptotic cell death by ebselen, a glutathione peroxidase mimic. J Cardiovasc Pharmacol. 2000; 36:601-8.

18. Suzuki K, Kostin S, Person V, Elsasser A, Schaper J. Time course of the apoptotic cascade and effects of caspase inhibitors in adult rat ventricular cardiomyocytes. J Mol Cell Cardiol. 2001;33:98394.

19. Ricci JE, Gottlieb RA, Green DR. Caspase-mediated loss of mitochondrial function and generation of reactive oxygen species during apoptosis. J Cell Biol. 2003;160:65-75. 